\title{
Improved Measurement Method of Circularly-Polarized Antennas Based on Linear-Component Amplitudes
}

\author{
Daoyu Wang, Min Wang*, Nuo Xu, Wen Wu \\ JGMT Ministerial Key Laboratory, Nanjing University of Science and Technology, Nanjing, China \\ Email: *wangmin@mail.njust.edu.cn
}

How to cite this paper: Wang, D.Y., Wang, M., Xu, N. and $\mathrm{Wu}, \mathrm{W}$. (2017) Improved Measurement Method of CircularlyPolarized Antennas Based on Linear-Component Amplitudes. Open Journal of Antennas and Propagation, 5, 36-45.

https://doi.org/10.4236/ojapr.2017.51004

Received: January 26, 2017

Accepted: March 24, 2017

Published: March 27, 2017

Copyright (c) 2017 by authors and Scientific Research Publishing Inc. This work is licensed under the Creative Commons Attribution International License (CC BY 4.0).

http://creativecommons.org/licenses/by/4.0/

\begin{abstract}
An improved measurement method of circularly-polarized (CP) antennas based on linear-component amplitudes is proposed in this paper. By utilizing two sets of orthogonal linear polarization (LP) amplitudes, measurement on axial ratio (AR) of $\mathrm{CP}$ antennas can be realized without phase information. However, the rotation sense of the co-polarization cannot be determined due to the absence of the phase information. Above problem is discussed here for the first time, and a solution is presented to determine the rotation sense of the co-polarization by using common auxiliary $\mathrm{CP}$ antennas. In addition, there will be some particular cases with large errors in actual measurement. Here a corresponding solution method is given. Finally, co-polarization and cross-polarization patterns can be further obtained from AR results. To verify this improved method, a self-developed CP microstrip array was measured. The measured results are in agreement with the simulated results, which prove this method is correct, effective and practical.
\end{abstract}

\section{Keywords}

Circularly-Polarized Antenna, Measurement Method, Linear-Component Amplitudes, Measurement Improvement

\section{Introduction}

Circularly-polarized (CP) antennas have many advantages such as insensitivity to polarization locations, elimination of the signal Faraday rotation effect caused by the ionosphere, and strong anti-interference ability. Therefore, they are widely used in satellite communication, radar, GPS and other systems. It is very important to measure their characteristics of axial ratio (AR), rotation sense, pattern and so on. By means of the measurement based on circular components, it is 
easy to measure CP antennas [1]. However, the auxiliary CP antennas with high polarization purity are rare in reality, so the uncertainty of this method is relatively large. While linearly-polarized antennas can achieve high polarization isolation easily, so it's more effective to study the method of measuring the $\mathrm{CP}$ antenna based on linear components.

Two methods about measuring ARs of CP antennas based on linear components are discussed in [2]. The first method is to measure one set of orthogonal LP amplitudes and phases by utilizing linearly-polarized auxiliary antennas. Another method is to measure only two sets of orthogonal LP amplitudes without phase measurement. ARs and patterns are separately obtained in [3] [4] [5] by using the first method. However, the phase measurement is limited by measuring equipment and the error is relatively large in fact. The characteristics of ARs are got by utilizing the second method in [6] and [7]. It is not necessary to measure phase information, while amplitudes can be measured accurately. As a result, the measuring accuracy of $A R$ is improved. But this method also has a problem that the rotation sense of the co-polarization cannot be determined due to the absence of the phase information. There is no analysis about this problem in the related records.

An improved measurement method of CP antennas based on linear-component amplitudes is proposed in this paper. It is the first time to point out the problem that the rotation sense of the co-polarization cannot be determined. And a solution is presented to determine the rotation sense by using common $\mathrm{CP}$ auxiliary antennas. In addition, some particular cases with large errors occur in practical measurement, here revises and improvements are given. Finally, copolarization and cross-polarization patterns are further obtained from AR results. To verify this improved method, a self-developed CP microstrip array was measured repeatedly. The measured results are in agreement with the simulated results, which prove the correction method is correct, effective and practical.

\section{Measurement Based on Linear-Component Amplitudes and Phases}

The polarization state of the electromagnetic wave is distinguished by the orientation of its electric field vector. In the propagation direction of electromagnetic wave, the electric field vector moves around a circle. According to the orbit of the vector, the electromagnetic waves can be divided into linearly-polarized waves, circularly-polarized waves and elliptically-polarized waves. The transverse electromagnetic wave of antenna radiation in the far field is called plane polarized wave, and its arbitrary polarization state is elliptical polarization, as shown in Figure 1. The AR is defined as the ratio of the major axis $2 A$ and the minor axis $2 B$ of the polarization ellipse, denoted as $\mathrm{AR}=2 A / 2 B$. While $A=B, \mathrm{AR}=$ 1 and it is expressed as circular polarization. While $A \neq 0$ and $B=0, \quad \mathrm{AR}=\infty$ and it is expressed as linear polarization. It can be seen that linear polarization and circular polarization are two particular cases of elliptical polarization [8].

The elliptically-polarized wave can be decomposed into two orthogonal linearly- 
polarized waves [9]. As shown in Figure 1, for arbitrary electric field vector $E$, it can be decomposed into two orthogonal components $E_{X}$ along $X$-axis and $E_{y}$ along $y$-axis. For the plane with $z=0, E_{x}$ and $E_{y}$ can be expressed as:

$$
\begin{gathered}
E_{x}=E_{1} \sin (\omega t) \\
E_{y}=E_{2} \sin (\omega t+\delta)
\end{gathered}
$$

where in $E_{1}$ and $E_{2}$ are the horizontally-polarized amplitude and the verticallypolarized wave amplitude respectively. $\delta$ is the phase difference between $E_{1}$ and $E_{2}$.

In Figure 1, $u$-axis and $v$-axis coincide with major axis and minor axis of polarization ellipse. So the semi-major axis $A$ and semi-minor $B$ can be expressed as [10]:

$$
\begin{aligned}
& A=\sqrt{\frac{1}{2}\left(E_{1}^{2}+E_{2}^{2}+\sqrt{E_{1}^{4}+E_{2}^{4}+2 E_{1}^{2} E_{2}^{2} \cos (2 \delta)}\right) .} \\
& B=\sqrt{\frac{1}{2}\left(E_{1}^{2}+E_{2}^{2}-\sqrt{E_{1}^{4}+E_{2}^{4}+2 E_{1}^{2} E_{2}^{2} \cos (2 \delta)}\right) .}
\end{aligned}
$$

$\tau$ is the inclination of the polarization ellipse with respect to $\varphi=0^{\circ}$ and its expression is:

$$
\tau=\frac{1}{2} \arctan \frac{2 E_{1} E_{2} \cos \delta}{E_{1}^{2}-E_{2}^{2}} .
$$

Comprehensive above, by measuring one set of orthogonal amplitudes $E_{1}, E_{2}$ and phase difference $\delta$ with a linearly-polarized antenna, the AR can be obtained as follows:

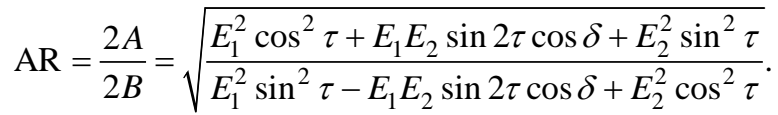

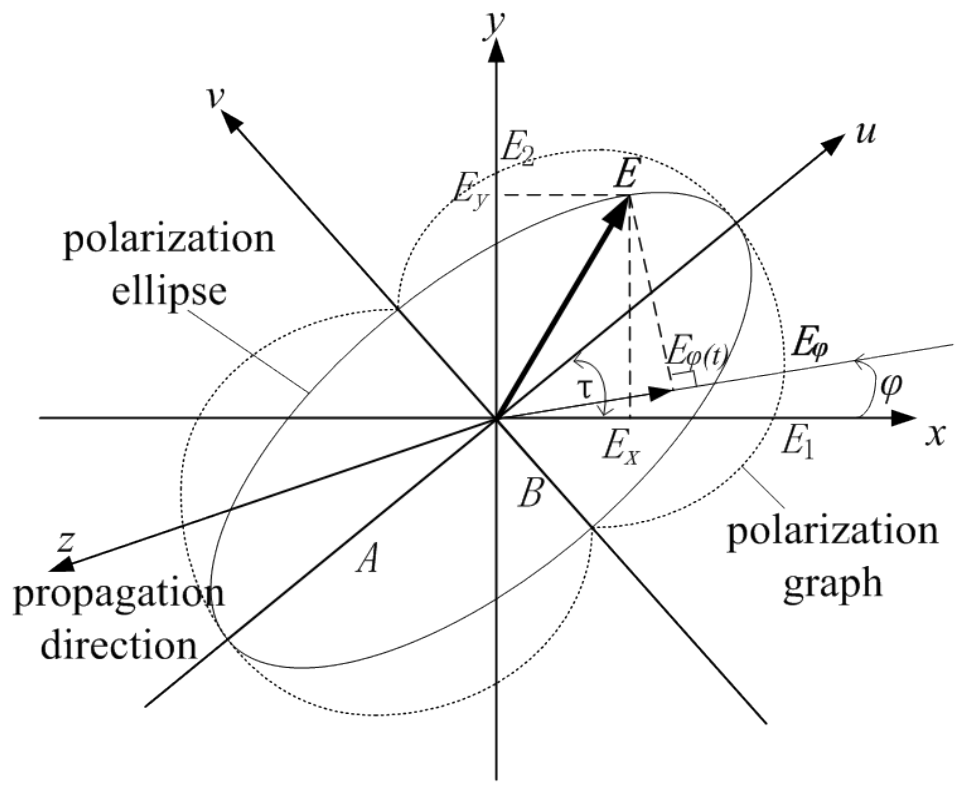

Figure 1. Elliptically polarized wave. 
The rotation sense of the elliptically-polarized wave can be determined by $\delta$. It is left-handed circular polarization when $0^{\circ}<\delta<180^{\circ}$, while it is right-handed circular polarization when $-180^{\circ}<\delta<0^{\circ}$.

In general antenna test environment, usually it is difficult to obtain accurate phase information. However, accurate measurement on amplitude is much easier to implement, so it has unique significance to study the measurement on AR only based on linear-component amplitudes.

\section{Measurement Only Based on Linear-Component Amplitudes}

\subsection{Derivation of Phase Information}

AR cannot be obtained by Equations (5) and (6) when only using two linearcomponent amplitudes without phase information. Here a derivation of phase is given from four linear-component amplitudes.

In the $x y$ plane, as shown in Figure 1, the projection of electric field vector $E$ in arbitrary polarization direction of $\varphi$ can be expressed as:

$$
E_{\varphi}(t)=E_{x} \cos \varphi+E_{y} \sin \varphi .
$$

Equations (1) and (2) are substituted into the above equation and the following equation can be derived:

$$
E_{\varphi}(t)=E_{\varphi} \sin (\omega t+\gamma)
$$

where in

$$
E_{\varphi}^{2}=\frac{1}{2}\left[E_{1}^{2}+E_{2}^{2}+\left(E_{1}^{2}-E_{2}^{2}\right) \cos 2 \varphi+2 E_{1} E_{2} \sin 2 \varphi \cos \delta\right] .
$$

Equation (9) gives the relationship between $E_{\varphi}$ and $\varphi$, and its corresponding graph is called polarization graph, as shown in Figure 1. The graph gives the maximum projection of $E$ in the direction of $\varphi$. Actually, $E_{\varphi}$ is the field response of the linearly-polarized antenna rotating to the direction of $\varphi$ in the $x y$ plane. The maximum and minimum values of the polarization graph coincide with the maximum and minimum values of the polarization ellipse, respectively.

Given two sets of arbitrary orthogonal linear-component amplitudes, phase information can be obtained when they are substituted into Equation (9). For example, through rotating linearly-polarized antenna, two sets of orthogonal LP amplitudes $E_{1}, E_{2}, E_{3}, E_{4}$ at $\varphi=0^{\circ}, 90^{\circ}, 45^{\circ}, 135^{\circ}$ can be obtained, phase can be derived by Equation (9), as follows:

$$
\cos \delta=\frac{E_{3}^{2}-E_{4}^{2}}{2 E_{1} E_{2}}
$$

\subsection{Derivation of AR}

By substituting Equation (10) into Equations (5) and (6), $\tau$ and AR can be obtained:

$$
\tau=\frac{1}{2} \arctan \frac{E_{3}^{2}-E_{4}^{2}}{E_{1}^{2}-E_{2}^{2}}
$$




$$
\mathrm{AR}=\sqrt{\frac{E_{1}^{2} \cos ^{2} \tau+\frac{1}{2}\left(E_{3}^{2}-E_{4}^{2}\right) \sin 2 \tau+E_{2}^{2} \sin ^{2} \tau}{E_{1}^{2} \sin ^{2} \tau-\frac{1}{2}\left(E_{3}^{2}-E_{4}^{2}\right) \sin 2 \tau+E_{2}^{2} \cos ^{2} \tau} .} .
$$

Equation (12) is the formula about measuring AR by utilizing only four amplitudes. Also because of the principle that total power of arbitrary two orthogonal components is the same:

$$
E_{3}^{2}+E_{4}^{2}=E_{1}^{2}+E_{2}^{2}
$$

it can be seen that $E_{4}$ is redundant. So sometimes, only three amplitudes $E_{1}, E_{2}$, $E_{3}$ have to be measured. However, the measurement accuracy can be controlled by utilizing four amplitudes. Therefore, it is better to use the method by utilizing four amplitudes.

\subsection{Determination of the Rotation Sense of Co-Polarization}

Phase information represented by Equation (10) can be obtained from above derivation. However, the range of $\operatorname{arcos} \delta$ is $(0, \pi)$, so the range of $\delta$ cannot be determined whether it is $\left(0^{\circ}, 180^{\circ}\right)$ or $\left(-180^{\circ}, 0^{\circ}\right)$. This means that the method loses part of phase information, so the rotation sense of co-polarization cannot be determined. This problem has not been mentioned in the related records.

Here a determination method is given by adopting two $\mathrm{CP}$ auxiliary antennas with identical structure but reversed rotation senses. Two auxiliary antennas are separately used to measure the amplitude of the antenna to be measured. Then the rotation sense of the co-polarization is the rotation sense of the auxiliary antenna which can measure larger amplitude. It is unnecessary to adopt the $\mathrm{CP}$ auxiliary antenna with high polarization purity used in the measurement based on circular components. Here CP auxiliary antennas with general performance are used and they are easy to be implemented in the actual situation.

\subsection{Treatment of Particular Cases in Measurement}

When the electric field wave approaches linear polarization in particular directions, there will be particular cases with large errors in the measurement. Four kinds of LP cases in particular directions are shown in Figure 2. And their values of normalized amplitudes, $\delta$ and $\tau$ are recorded in Table 1.

For the two cases in Figure 2(a) and Figure 2(b), the numerator and denominator of Equation (10) are all close to 0. It causes relatively large errors since $|\cos \delta|$ may be greater than 1. Although $\tau$ can be stably solved from Equation (11), errors are still large. However, for the two cases in Figure 2(c) and Figure 2(d), $|\cos \delta|$ can be solved accurately. Thus for the two cases in Figure 2(a) and Figure 2(b), $\cos \delta^{\prime}$ can be accurately obtained from Figure 2(c) and Figure 2(d). Let $E_{1}^{\prime}=E_{3}, \quad E_{2}^{\prime}=E_{4}, \quad E_{3}^{\prime}=E_{2}, \quad E_{4}^{\prime}=E_{1}$, then

$$
\cos \delta^{\prime}=\frac{E_{3}^{\prime 2}-E_{4}^{\prime 2}}{2 E_{1}^{\prime} E_{2}^{\prime}}=\frac{E_{2}^{2}-E_{1}^{2}}{2 E_{3} E_{4}} .
$$




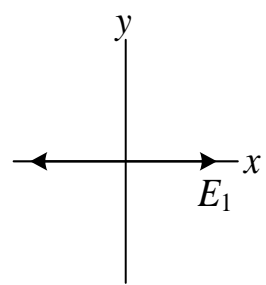

(a)

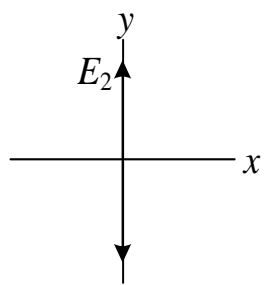

(b)

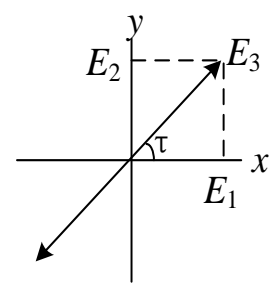

(c)

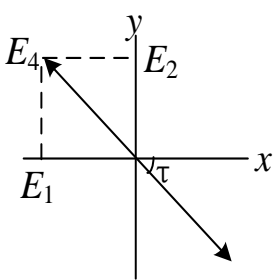

(d)

Figure 2. LP cases in particular directions.

Table 1. Normalized amplitudes, $\delta$ and $\tau$ values of LP cases.

\begin{tabular}{ccccccc}
\hline Figure 2 & $E_{1}$ & $E_{2}$ & $E_{3}$ & $E_{4}$ & $\delta$ & $\tau$ \\
\hline (a) & 1 & 0 & $\sqrt{2} / 2$ & $\sqrt{2} / 2$ & $/$ & 0 \\
(b) & 0 & 1 & $\sqrt{2} / 2$ & $\sqrt{2} / 2$ & $/$ & $\pi / 2$ \\
(c) & $\sqrt{2} / 2$ & $\sqrt{2} / 2$ & 1 & 0 & 0 & $\pi / 4$ \\
(d) & $\sqrt{2} / 2$ & $\sqrt{2} / 2$ & 0 & 1 & $\pi$ & $-\pi / 4$ \\
\hline
\end{tabular}

$$
\begin{gathered}
\tau^{\prime}=\frac{1}{2} \arctan \frac{E_{2}^{2}-E_{1}^{2}}{E_{3}^{2}-E_{4}^{2}} . \\
\mathrm{AR}=\sqrt{\frac{E_{3}^{2} \cos ^{2} \tau^{\prime}+\frac{1}{2}\left(E_{2}^{2}-E_{1}^{2}\right) \sin 2 \tau^{\prime}+E_{4}^{2} \sin ^{2} \tau^{\prime}}{E_{3}^{2} \sin ^{2} \tau^{\prime}-\frac{1}{2}\left(E_{2}^{2}-E_{1}^{2}\right) \sin 2 \tau^{\prime}+E_{4}^{2} \cos ^{2} \tau^{\prime}}} .
\end{gathered}
$$

In practice, the particular case of $E_{1} \approx E_{2} \approx E_{3} \approx E_{4}$ appears occasionally. In this case, performance of circular polarization is pretty good. However, the numerator and denominator of Equation (11) are all close to $0, \tau$ has a large error as a result. While $\delta$ approximately equals to $\pm 90^{\circ}$ by utilizing Equation (10), then AR can be accurately obtained through dividing Equation (3) by Equation (4).

\subsection{Derivation of Patterns}

Co-polarization and cross-polarization can be obtained from AR results as follows. The elliptically-polarized wave can also be decomposed into two orthogonal $\mathrm{CP}$ waves with reversed rotation senses. For arbitrary electric field vector $E$, it can be decomposed into right-handed CP component $\boldsymbol{E}_{R}$ and left-handed CP component $\boldsymbol{E}_{L}$. If $E_{R}>E_{L}$, the right-hand CP component is co-polarization component and the left-hand $\mathrm{CP}$ component is cross-polarization component, otherwise the opposite. $E_{R}$ and $E_{L}$ satisfy the following relations:

$$
E_{L}^{2}+E_{R}^{2}=E_{1}^{2}+E_{2}^{2} \text {. }
$$

AR can also be expressed by $E_{R}, E_{L}$ (suppose that $E_{R}>E_{L}$ ):

Let $\Gamma=\frac{E_{L}}{E_{R}}$, then

$$
\mathrm{AR}=\frac{E_{R}+E_{L}}{E_{R}-E_{L}} \text {. }
$$

$$
\Gamma=\frac{\mathrm{AR}-1}{\mathrm{AR}+1} .
$$


Substitute $\Gamma$ into Equation (17), then

$$
\begin{gathered}
E_{R}=\sqrt{\frac{\left(E_{1}^{2}+E_{2}^{2}\right)}{1+\Gamma^{2}}} . \\
E_{L}=\sqrt{\frac{\Gamma^{2}\left(E_{1}^{2}+E_{2}^{2}\right)}{1+\Gamma^{2}}} .
\end{gathered}
$$

Therefore, patterns of the antenna to be measured can be represented by (20) and (21), corresponding to the co-polarization component and the cross-polarization component, respectively.

\section{Measurement Verification}

A self-developed CP microstrip array shown in Figure 3 was measured repeatedly by using the improved method. The CP antenna to be measured in Figure 3 is used as a receiving antenna and rotates with the turntable. And the linearlypolarized double-ridged horn shown in Figure 4 is kept still as a transmitting antenna.

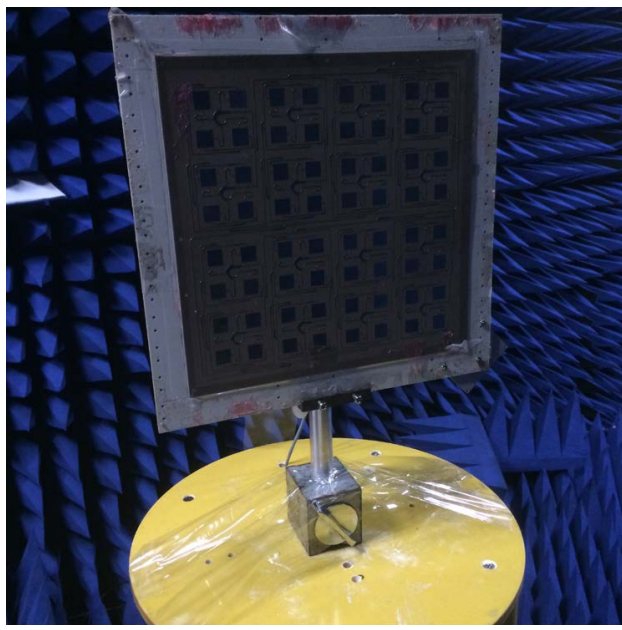

Figure 3. Self-developed CP antenna.

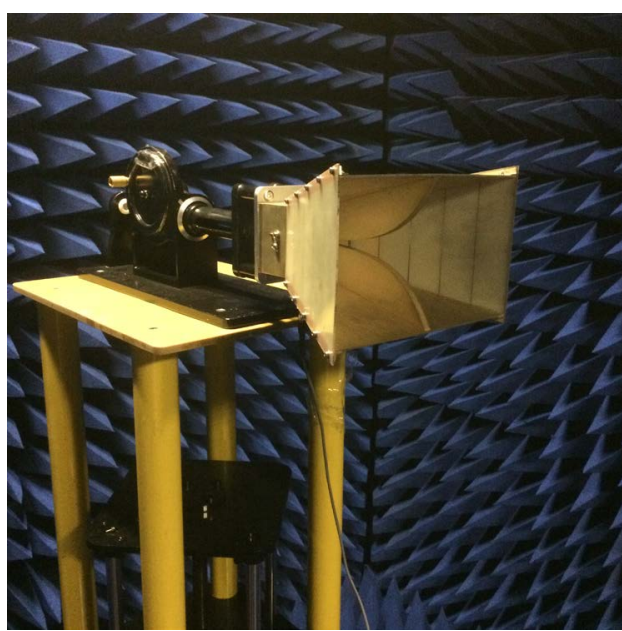

Figure 4. Linearly-polarized double-ridged horn. 
The polarization direction of the linearly-polarized transmitting antenna is separately rotated at $0^{\circ}, 90^{\circ}, 45^{\circ}$ and $135^{\circ}$, and four linearly-polarized amplitudes can be measured quickly and accurately. Then ARs and patterns can be solved from above derivations based on the amplitudes conveniently. The measured results are compared with the simulation results, as shown in Figures 5-7. The simulation results are derived from Ansoft HFSS.

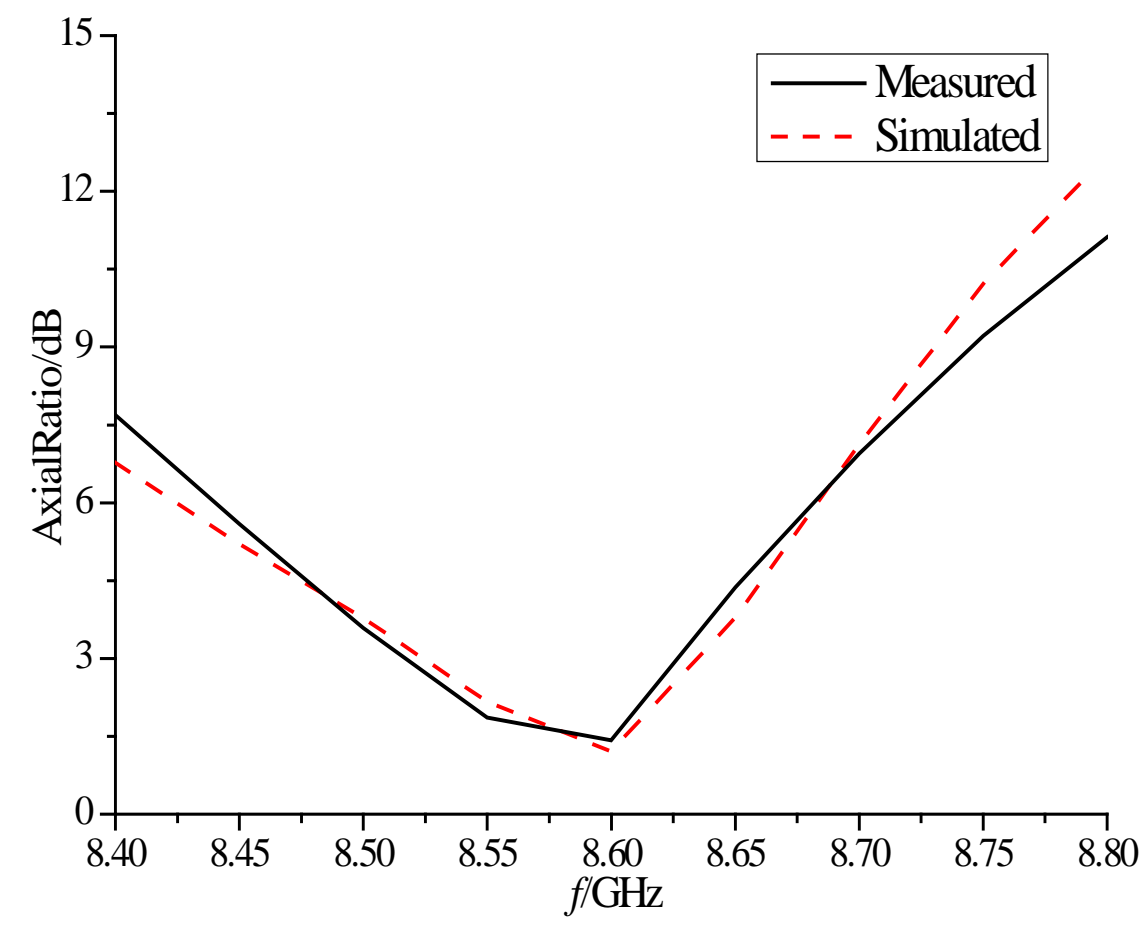

Figure 5. Curves of ARs versus frequency $\left(\varphi=0^{\circ}, \theta=0^{\circ}\right)$.

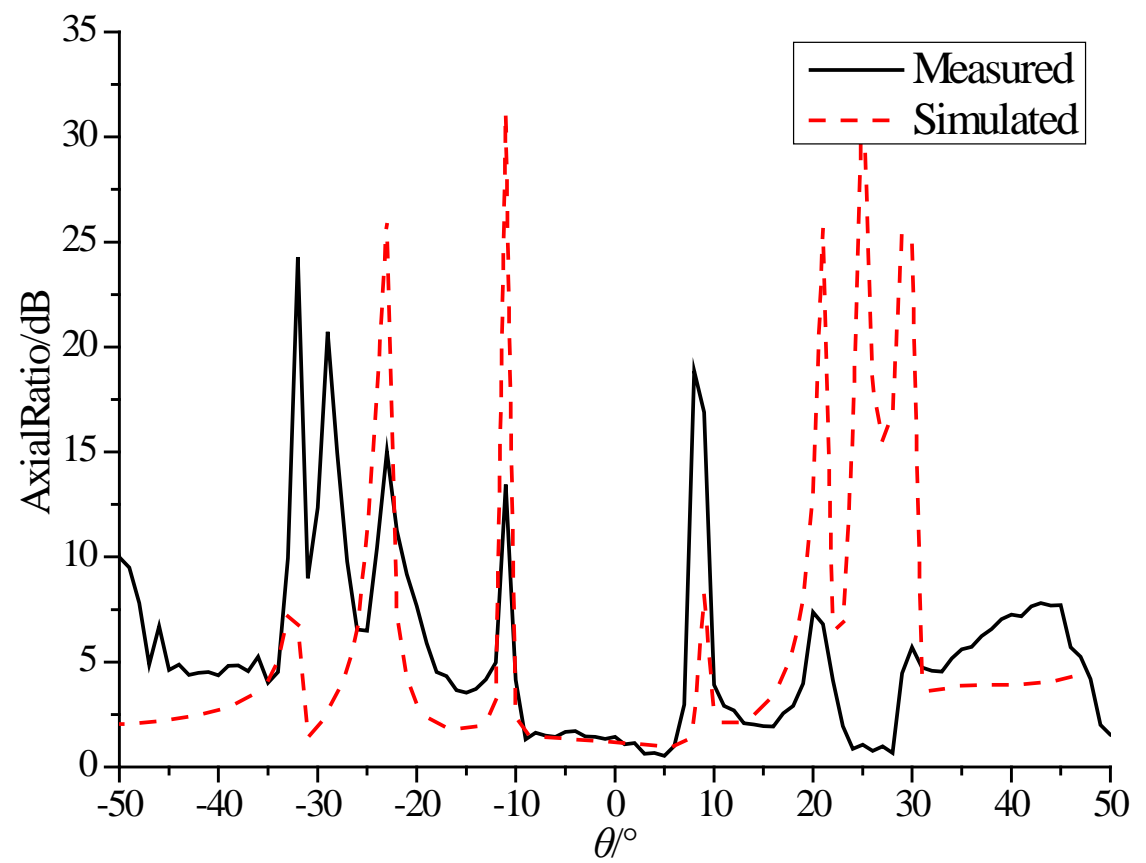

Figure 6. Curves of ARs versus $\theta$ at center frequency of $8.6 \mathrm{GHz}\left(\varphi=0^{\circ}\right)$. 


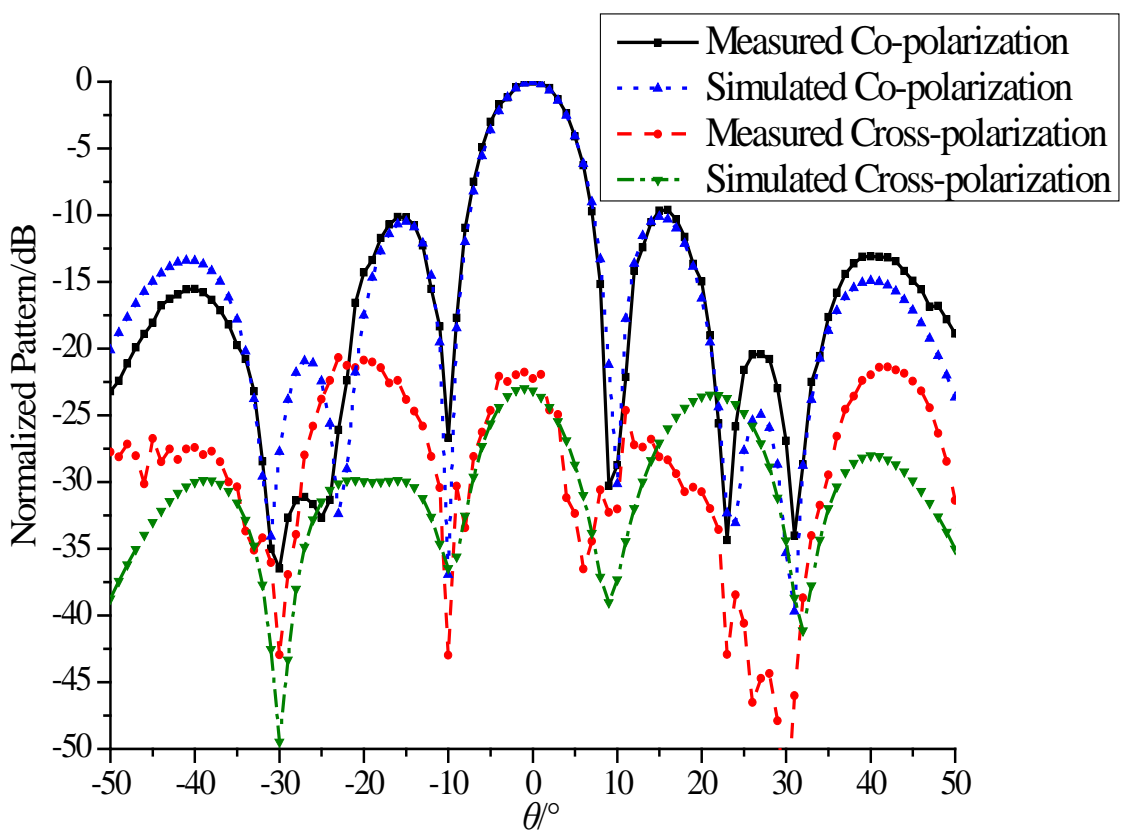

Figure 7. Normalized patterns at center frequency of $8.6 \mathrm{GHz}\left(\varphi=0^{\circ}\right)$.

Measured results in Figure 5 show that the AR curves versus frequency agree very well with the simulated one from $8.47 \mathrm{GHz}$ to $8.68 \mathrm{GHz}$. The minima are both about $1.5 \mathrm{~dB}$ at the center frequency of $8.6 \mathrm{GHz}$. There are only some certain differences at lower or upper frequencies, which can be attributed to the machining tolerance. At the center frequency of $8.6 \mathrm{GHz}$, measured and simulated curves of ARs versus $\theta$ at $\varphi=0^{\circ}$ also maintain good consistency in Figure 6. By utilizing common auxiliary antennas with determined rotation senses, the antenna to be measured is identified as a left-hand CP antenna. The measured and simulated normalized patterns shown in Figure 7 also match well. In patterns, when the LP case occurs, the co-polarization component is approximately equal to the cross-polarization component, which causes that two components cannot be distinguished. This is the limitation of the proposed work. However, in this case the amplitudes of two components both are small and slight effect occurs on the overall performance of the patterns. It can be solved with nearest distribution according to the smoothness of the curves. Two components of other parts in patterns are clear and can be distinguished easily.

To sum up, it can be seen that the improved method is correct, practical and effective.

\section{Conclusion}

A method for measuring characteristic parameters of $\mathrm{CP}$ antennas is discussed based on linear-component amplitudes in this paper. By measuring two arbitrary sets of orthogonal LP amplitudes, the ARs can be obtained quickly and conveniently. However, the rotation sense of co-polarization cannot be determined in original measurement method, so here a corresponding solution is presented to determine it by using common CP auxiliary antennas. Also the particular cases 
in the measurement are considered and perfect processing method has been established. The revised and improved method has the advantages of accuracy, convenience and efficiency. It can meet the basic demand of scientific research and engineering for $\mathrm{CP}$ antenna measurement.

\section{Grant Information}

This work was supported by National Natural Science Foundation of China under Grant 61401208.

\section{References}

[1] Zhang, X.P. (2006) The Study on Compensated Compact Range (CCR) Antenna Measurement Technology. Spacecraft Environment Engineering, 23, 321-328. http://doi.org/10.3969/j.issn.1673-1379.2006.06.003

[2] Lin, C.L. (1987) Antenna Measurement Technique. Chengdu Telecommunication Engineering College Press, Chengdu.

[3] Toh, B.Y., Cahill, R. and Fusco, V.F. (2003) Understanding and Measuring Circular Polarization. IEEE Transaction on Education, 46, 313-318. http://doi.org/10.1109/TE.2003.813519

[4] Shang, J.P., Fu, D.M., Jiang, S. and Deng, Y.B. (2009) Method for Measuring the Characteristic Parameter of the Circular Polarization Antenna. Journal of Xidian University (Natural Science), 36, 106-110. http://doi.org/10.3969/j.issn.1001-2400.2009.01.020

[5] Guo, J. and Wan, S.S. (2009) Measuring Method for Circular Polarization Antennas in Far Field. Journal of Nanjing University of Aeronautics \& Astronautics, 41, 62 66. http://doi.org/10.3969/j.issn.1005-2615.2009.z1.014

[6] Li, N.J., Feng, Y.L., Wang, J.F. and Dang, J.J. (2013) Fast Measuring Axial Ratio of Circular Polarization Antennas Based on Linear Polarization Antenna. Infrared and Laser Engineering, 42, 2216-2220.

[7] Zhang, X.P. and Zhou, H.A. (2009) An Accurate Test Method for Circular Polarized Antenna. Spacecraft Environment Engineering, 26, 67-70. http://doi.org/0.3969/j.issn.1673-1379.2009.01.016

[8] Qin, S.Y., Yang, K.Z. and Chen, H. (2003) Measuring Technique of Gain for Different Polarization Antenna. Journal of Electronic Measurement and Instrumentation, 17, 7-11. http://doi.org/10.13382/j.jemi.2003.01.002

[9] Yu, J. and Zhao, J. (2014) Analyze the Gain Results of Polarized Antenna with Different Method in Anechoic Chamber. Foreign Electronic Measurement Technology, 33, 34-36. http://doi.org/10.3969/j.issn.1002-8978.2014.09.010

[10] Fang, D.G. (2010) Antenna Theory and Microstrip Antennas. CRC Press, Boca Raton. 
Submit or recommend next manuscript to SCIRP and we will provide best service for you:

Accepting pre-submission inquiries through Email, Facebook, LinkedIn, Twitter, etc. A wide selection of journals (inclusive of 9 subjects, more than 200 journals)

Providing 24-hour high-quality service

User-friendly online submission system

Fair and swift peer-review system

Efficient typesetting and proofreading procedure

Display of the result of downloads and visits, as well as the number of cited articles Maximum dissemination of your research work

Submit your manuscript at: http://papersubmission.scirp.org/

Or contact ojapr@scirp.org 